\title{
Study on Wear and Morphological Behavior of Electron Beam Dose Irradiated Polyoxymethylene Copolymer (POM-C)
}

\author{
Md. Shahinur Rahman ${ }^{1 *}$, Heon- Ju Lee ${ }^{1}$, Jong-Keun Yang ${ }^{1}$, Konstantin Lyakhov $^{1}$ and \\ Muhammad Athar Uddin ${ }^{2}$ \\ ${ }^{1}$ Department of Nuclear and Energy Engineering, Jeju National University, \\ Jeju 63243, Republic of Korea. \\ ${ }^{2}$ Department of Electrical and Electronic Engineering, International Islamic \\ University, Chittagong, Bangladesh.
}

*Corresponding author: shahineee33@gmail.com

\begin{abstract}
Polyoxymethylene copolymer (POM-C) is the most prominent engineering thermoplastic consisting of repeating carbon-oxygen bonds in the form of oxymethylene groups $\left(\mathrm{OCH}_{2}\right)$. It is widely used to make small gear wheels, ball bearings, precision parts, automotive and consumer electronics. In this study, the POM-C round blocks were irradiated with $165 \mathrm{KeV}$ electron beam energy in five doses $(100,200,300,500$ and 700 $\mathrm{kGy}$ ) in vacuum condition at room temperature. The wear rate, surface hardness and morphological properties of electron beam dose irradiated POM-C blocks surfaces have been analyzed using pin on disk tribometer, optical microscopy, nano-indenter, Raman spectroscopy, 3D nano surface profiler and scanning electron microscopy (SEM). The electron beam irradiation transferred the wear phenomena of unirradiated POM-C sample from the abrasive wear (plough and cracks), adhesive wear (grooving/striation, micropitting) and scraping to mild scraping and striation for the $100 \mathrm{kGy}$ dose irradiated POM-C sample due to cross-linking (macroscopic networks), chemical free radicals formations and partial physical modification (smoothness), which can be concluded from tribometer, optical microscopic, SEM and Raman spectroscopic observations. It also reduced the surface wear rate and average surface roughness with increasing microsurface hardness at threshold value of cross-linking among all unirradiated and others doses irradiated POM-C blocks. The level of tribological (wear and morphology) attribute improvement relies on the electron beam irradiation condition (energy and dose rate) depending on chemical and physical factors of polymeric materials.
\end{abstract}

Keywords: Electron- beam; Wear- rate; Surface -hardness; Raman- spectroscopy; Surface- roughness; SEM.

\section{INTRODUCTION}

Polyoxymethylene copolymer (POM-C) is a superior engineering thermoplastic for its owned outstanding chemical, physical, self lubricating and mechanical properties [1]. However, poor wear resistance and severe crack formation are real drawbacks of POM-C in applications of high tech areas. Radiation induced surface modification of polymeric materials is a prominent and cutting edge new method to solve this intricate problem [2 5]. Electron beam irradiation on pure polymeric materials results in the formation of three-dimensionally bonded networks through the chemical free radicals generation [2]. The generated free radicals can initiate complex reactions which can lead to cross-linking or chain scission of polymer chains. As a result, radiation changes the chemical, physical, electrical, mechanical, optical and morphological properties of irradiated polymeric 
materials. The radiation initiated changes can occur in crystalline and amorphous region of polymeric materials through cross-linking and chain scission depending on chemical and physical factors of irradiated materials [6 - 8].

Recently electron beam irradiation method is becoming an optimistic approach to optimize the physical, mechanical and chemical properties of polymeric materials. Basically Electron beam (EB) irradiated energy intercepts the polymeric surface materials and, it can change in molecular structural arrangement through ionization, atomic displacement, carbonization and free radicals generation $[2,8]$. The EB radiation not only changes the chemical and physical factors of polymeric materials, rather it can also increase the trapped charges or make some defects in the polymeric materials. The EB irradiation initiated well suited cross-linking can develop the physical and morphological properties of POM-C in molecular scale, which can reduce the wear and enhance the morphological properties in optimum level. The optimum improvement of wear loss in POM-C industrial applications can save huge amount of energy and GDP of any highly industrialized nation.

The general objective of this study is to reduce the abrasive and adhesive wear rate of POM-C with good correlation among chemical, physical and mechanical properties at optimum EB irradiation condition.

\section{EXPERIMENTAL}

The POM-C (1 mm thickness and $25 \mathrm{~mm}$ diameter) used was supplied from Korea Engineering Plastics Co. (Seoul, Korea). The density $-1.41 \mathrm{~g} / \mathrm{cm}^{3}$ (ISO 1183), melt flow rate $-3 \mathrm{~g} / 10 \mathrm{~min}$ and melting point $-165^{\circ} \mathrm{C}$ are the typical properties of used POM-C. EB irradiation was conducted under vacuum condition with an ELV-12 (coreless DC, induction coupling) electron accelerator at a voltage of $165 \mathrm{KeV}$ in KAPRA (Gangwondo, South Korea). The general diagram of ELV-12 is shown in Fig. 1. The POM-C samples (numbered as a-f) were irradiated to doses of 0, 100, 200, 300, 500 and $700 \mathrm{kGy}$, respectively. The EB current was $300 \mathrm{~mA}$ and the dose rate was $25 \mathrm{kGy}$ per pass. The worn surfaces morphology (abrasive and adhesive wear scars with crack formation) at tribometer oriented sliding tracks of unirradiated and irradiated POM-C blocks was observed with an Olympus BX51M computerized HR digital optical microscope. The dry sliding wear rate was observed on a J\&L Tech tribometer, operating in a ball on disk method. The SUJ 52100 steel ball slid against POM-C blocks rotating at a speed of 100 $\mathrm{mm} / \mathrm{s}$ unidirectionally over a period of 50 minutes $(300 \mathrm{~m})$. The applied normal load was $2 \mathrm{~N}$ with a Hertzian contact pressure of $102 \mathrm{MPa}$ during the dry sliding. The wear rate was calculated using the mass method (TSE 555 standard). The POM-C block surface micro-hardness test was carried out by a Hysitron TI 950 triboindenter at the nanoindentation depth of $1200 \mathrm{~nm}$. The surface morphology and chemical structure were observed using scanning electron microscope and Raman spectroscopy, respectively. The surface roughness of all unirradiated and irradiated POM-C samples was measured using 3D nano surface profiler (WT-250) at 20x objective. 


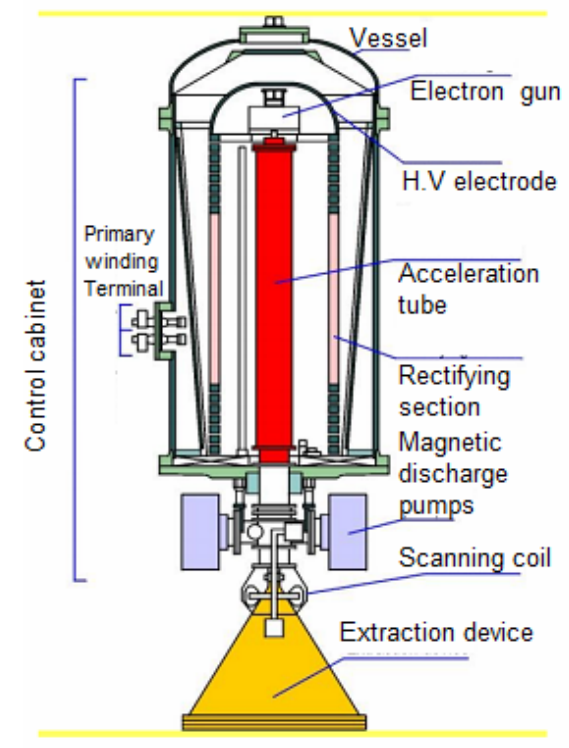

Fig. 1. General diagram of ELV-12 (KAPRA, Republic of Korea).

\section{RESULTS AND DISCUSSION}

\section{A. Wear rate and worn surfaces morphology}

The Optical microscopic images at x20 resolution in Fig. 2 (a-f) show the worn surfaces to analyze the wear mechanism at sliding tracks on POM-C blocks with and without EB dose irradiation. Variation in wear rate of POM-C blocks with different EB dose irradiation is shown in Fig. 3 (a -f). Wear is an intricate phenomenon which could reduce by different precise methods. The wear rate can vary with either change in micro-surface hardness or in surface chemical and physical conditions. The surface chemical and physical conditions can change with surface Electron beam (EB) dose delivered depending on chemical and physical factors of target sample. The wear rate for unirradiated POM-C block (a) is $2.65 \times 10^{-5} \mathrm{~mm}^{3} / \mathrm{Nm}$ shown in Fig. 3. The worn surface of unirradiated POM-C block is experienced with huge plastic ruffles and ripples flow and polymer deformation along the sliding track. It is also shrouded with grooving, scuffing, friction traces and crack formation perpendicular to sliding track shown in Fig. 2-a. In Fig. 2-b, the worn surface of $100 \mathrm{kGy}$ EB dose irradiated POM-C appears smooth with shallower and milder scuffing. It also indicates a significant decrease in wear rate (2 $\mathrm{x} 10^{-5} \mathrm{~mm}^{3} / \mathrm{Nm}$ ) compared to both unirradiated and others EB dose irradiated POM-C blocks shown in Fig. 3-b. It may due to carbonization, free radicals formation and surface increased micro-hardness [2-11]. In Fig. 2 (c-f), abrasive wear (plough and surface crack), polymer deformation, grooving, scuffing, flaws, huge fatigue cracks and ribbon tracks were increased with increasing surface dose delivered $(200-700 \mathrm{kGy})$. The increased surface dose delivered also increased the wear rate linearly shown in Fig. 3 (c - f), which may due to extravagant chain-scission. 
From the Optical microscopic, tribometer, SEM and 3D Nano surface profiler observations, it can be concluded that $165 \mathrm{KeV}, 100 \mathrm{kGy}$ EB dose irradiation transferred the wear behavior of POM-C block from the abrasive wear, adhesive wear and scraping for the unirradiated surface to shallow and mild scraping for the irradiated surface due to well suited cross-linking, partial physical degradation, carbonization and formation of a small molecular substance [12 - 14]. So, the $100 \mathrm{kGy}$ EB dose irradiated POM-C block surface treated as self- lubricating surface with decreasing surface wear rate during dry sliding condition.
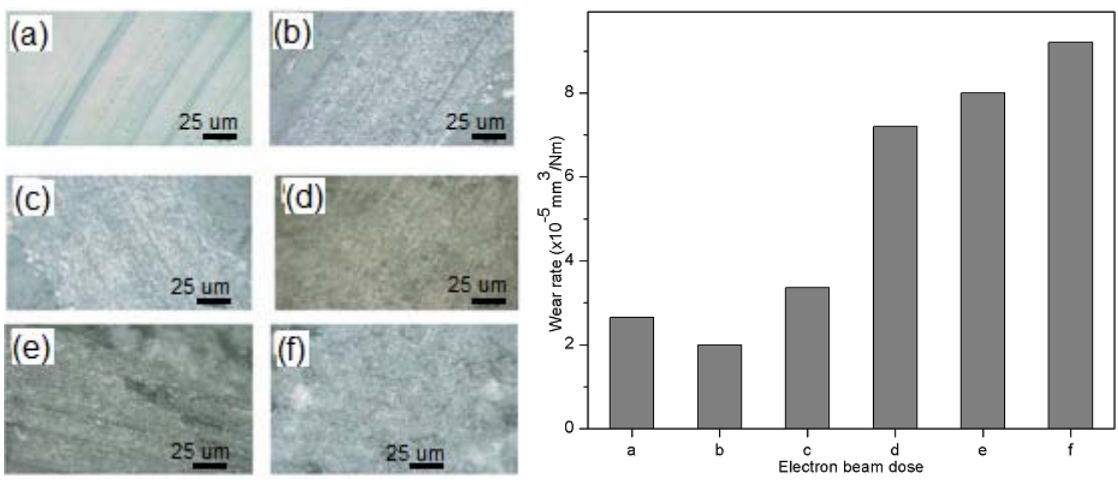

Fig. 2. Optical microscopic images of the worn surfaces at sliding tracks on POM-C surfaces with $\mathrm{x} 20$ resolution: (a) unirradiated POM-C; (b) 100 kGy; (c) 200 kGy; (d) $300 \mathrm{kGy}$; (e) $500 \mathrm{kGy}$ and (f) 700 kGy electron beam dose irradiated surfaces. Scale bar $25 \mu \mathrm{m}$ in each case.

Fig.3.Variation in wear rate with different EB dose irradiation $(0-700 \mathrm{kGy}$ numbered as a- $\mathrm{f}$ ).

\section{B. Surface hardness}

The average micro-surface hardness of different EB dose delivered POM-C blocks was carried out at $1200 \mathrm{~nm}$ indentation depth on three different locations. The micro-surface hardness can change by surface dose delivered induced cross-linking in polymeric materials depending on physical and chemical factors. The variation in micro-surface hardness for both unirradiated and EB dose delivered POM-C blocks is shown in Fig. 4 (a - $\mathrm{f}$ ). The micro surface hardness of unirradiated POM-C block surface is $180 \mathrm{MPa}$ shown in Fig. 4 -a. The $165 \mathrm{KeV}, 100 \mathrm{kGy}$ dose delivered surface exhibits higher micro-surface hardness $(220 \mathrm{MPa})$ compared to all unirradiated and other doses irradiated POM-C blocks shown in Fig. 4-b. It may attribute to the occurrence of radiation induced crosslinking, carbonization layer and structural changes, which could contribute to reduce the surface dry sliding wear rate shown in Fig. 5 [2 - 8, 11 - 17]. The cross-linking and carbonization phenomena are also ascertained from Raman spectroscopic observations. The average micro-surface hardness of POM-C blocks surfaces irradiated at 200, 300, 500 and $700 \mathrm{kGy}$ decrease linearly to $150,130,80$ and $60 \mathrm{MPa}$, respectively. The decrease in micro surface hardness also increase the surface wear rate shown in Fig. 5, which might be the reason of radiation induced chain scission and structural degradation [11 - 17]. 


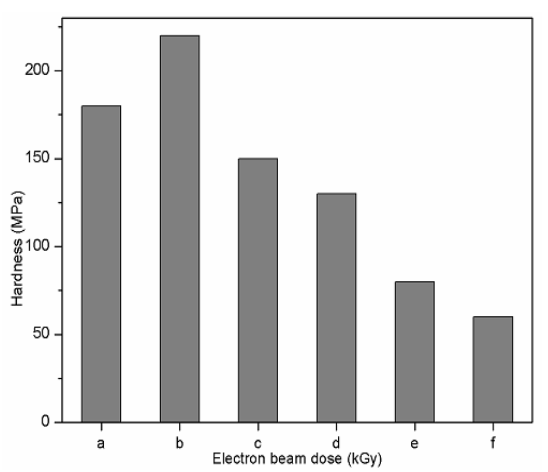

Fig. 4. Effect of EB dose on surface hardness $(\mathrm{MPa})$.

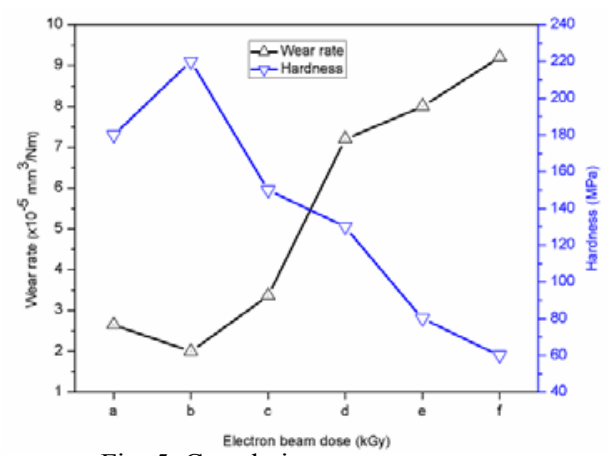

Fig. 5. Correlation among wear rate, hardness and EB dose.

\section{SEM analysis}

The EB dose irradiated and unirradiated POM-C samples were studied using scanning electron microscope to get better insights about surface morphology. Changes of surface morphology in different EB dose irradiation are noticed in Fig. 6 (a - f). In Fig. 6-a, some surface tracks and unevenness are observed on unirradiated POM-C sample. The $100 \mathrm{kGy}$ dose irradiated sample is smoother and fine compared to all unirradiated and irradiated surface shown in Fig. 6-b, which is due to well suited cross-linking and physical interactions between energetic electrons-target material atoms collisions [7 - 14]. A threshold value of cross-linking can initiate free radicals formation on the top surface by the absorption of radiated electron beam energy, which can easily improve the mechanical, morphological and chemical properties depending on chemical and physical factors of irradiated polymeric surface [2, 7 -9]. The threshold value of cross-linking mostly depends on the polymeric surface EB dose delivered. Cracking and ribbon traces are observed from 200 and 300 kGy EB dose irradiated POM-C surfaces shown in Fig. 6 (c-d). These cracking and ribbon traces can initiate through chain scission and, it can damage the polymeric surface through gas evolution, breaking polymeric molecular bonds and displacing the atomic arrangement. Severe cracking and high unevenness are observed in 500 and 700 kGy EB dose irradiated POM-C surfaces shown in Fig. 6 (e - f), which may be due to severe chain scission and gas evolution [7 - 9].

According to aforementioned observations, the $100 \mathrm{kGy}$ dose irradiation is well suited to get better morphology oriented surface. 

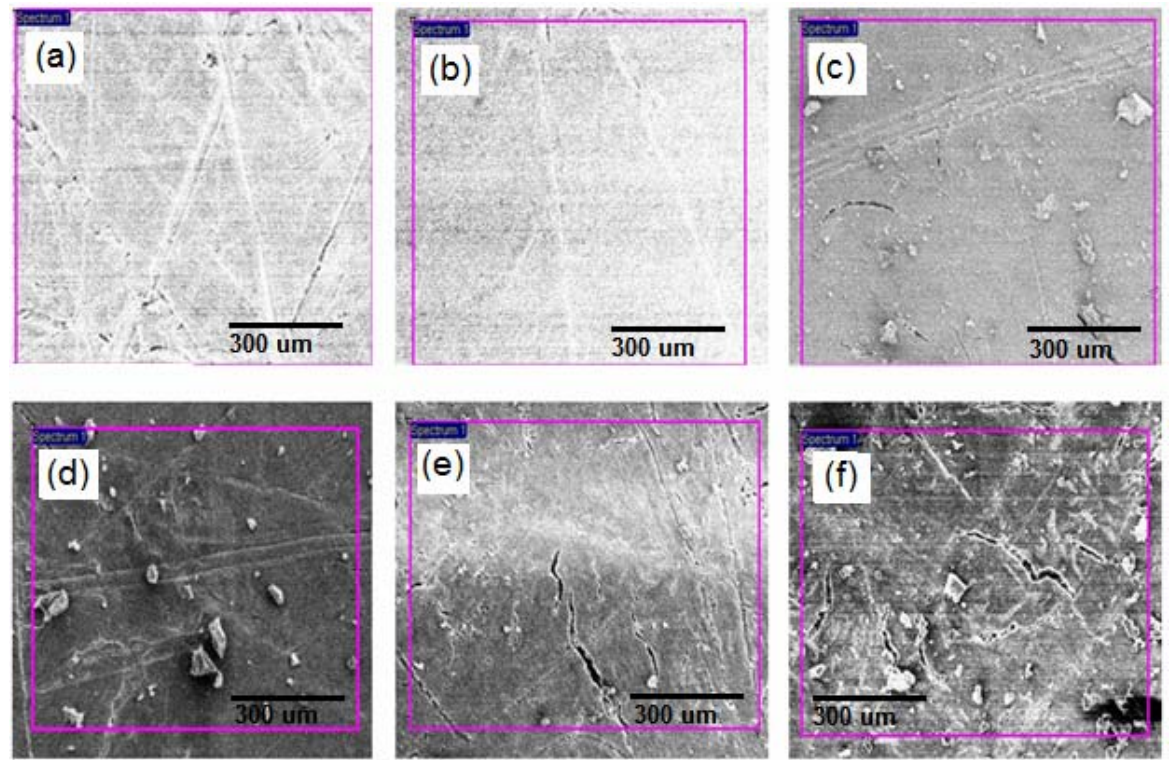

Fig. 6. SEM images: (a) unirradiated; (b) 100 kGy; (c) 200 kGy; (d) 300 kGy; (e) 500 kGy and (f) $700 \mathrm{kGy}$.

\section{Chemical structural analysis}

The chemical structural modification due to EB dose irradiation was studied using Raman spectroscopy (LABRAM HR EV) shown in Fig. 7 (a-f). Raman spectroscopy is also a viable tool to study about rotational and vibrational transitions in atomic level, which can give good insights for the degree of cross-linking as non-destructive technique [16 - 20]. The unirradiated POM-C sample gave the following vibrational peak assignments: 538 $\mathrm{cm}^{-1}(\delta, \mathrm{C}-\mathrm{O}-\mathrm{C}), 917 \mathrm{~cm}^{-1}\left(\mathrm{v}_{\mathrm{g}}, \mathrm{COC}\right), 1095 \mathrm{~cm}^{-1}$ ( $\left.\mathrm{v}_{\mathrm{a}}, \mathrm{C}-\mathrm{O}-\mathrm{C}\right), 1333 \mathrm{~cm}^{-1}\left(\mathrm{t}, \mathrm{CH}_{2}\right)$ and 1489 $\mathrm{cm}^{-1}\left(\delta, \mathrm{CH}_{2}\right)$ shown in Fig. 7-a [21]. The $100 \mathrm{kGy}$ EB dose irradiation highly increased the vibrational band strength and peak intensity of C-O-C symmetrical and asymmetrical stretching at 921 and $1104 \mathrm{~cm}^{-1}$, respectively. It also strengthens the vibrational band strength of $\mathrm{CH}_{2}$ bending at $1486 \mathrm{~cm}^{-1}$. These increased vibrational bands strength can protect form different chemical structural degradation due to well suited cross-linking shown in Fig. 7-b [16 - 21]. The others EB doses irradiation reduced the vibrational bands strength and peak intensity of all fundamental vibrational peak assignments gradually shown in Fig. 7 (c - f) due to increasing chain scission. So, $100 \mathrm{kGy}$ dose irradiation is well suited for the occurrence of appropriate cross-linking, which can give desired chemical structural modification. 


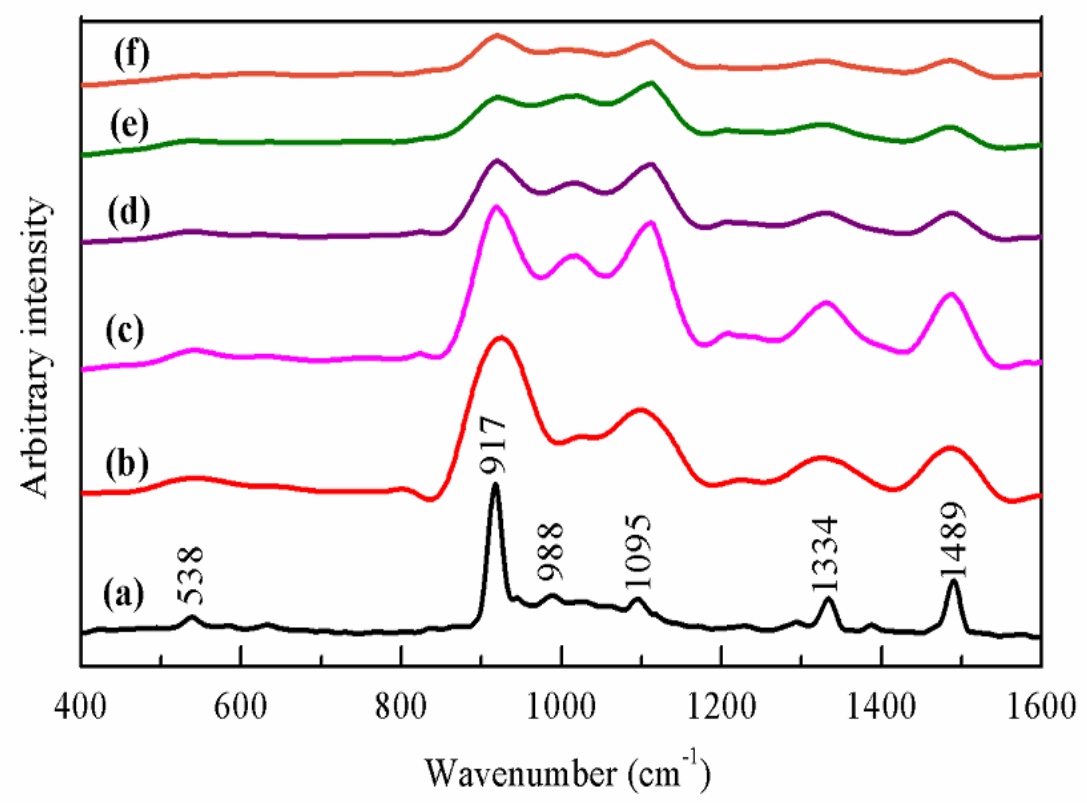

Fig. 7. Raman spectra demonstrating the chemical structural modification due to electron beam dose irradiation on POM-C surfaces: (a) unirradiated; (b) $100 \mathrm{kGy}$; (c) $200 \mathrm{kGy}$; (d) $300 \mathrm{kGy}$; (e) 500 kGy and (f) 700 kGy.

\section{E. Surface roughness analysis}

EB dose induced surface roughness changes are shown in Fig. 8 (a - f). The average surface roughness $(\mathrm{Ra})$ of unirradiated and $100 \mathrm{kGy}$ EB dose irradiated POM-C surfaces are $112.75 \mathrm{~nm}$ and $72.83 \mathrm{~nm}$, respectively. Significant surface roughness is noticed by 100 kGy EB dose irradiation, which can be the reason of energetic electrons- target atoms well controlled collisions and threshold value of cross-linking [2, $6-8]$. The threshold value of cross-linking can reduce surface roughness, when aggregation and atomic displacement are suppressed at desired level. In Fig. 8 (c -f), the 200, 300, 500 and 700 kGy EB dose irradiation increased the $\mathrm{R}_{\mathrm{a}}$ to $120.35,133.99,139.06$ and $205.40 \mathrm{~nm}$, respectively. The gradually increase of $R_{a}$ might be the reason of gradually increase chain scission depending on surface delivered EB dose [2 - 9, 16 - 20].

From the observations, it is clear that threshold value of cross-linking could reduce the average surface roughness depending on physical and chemical factors of target material surface, and it could reduce surface wear rate [2 - 8]. 


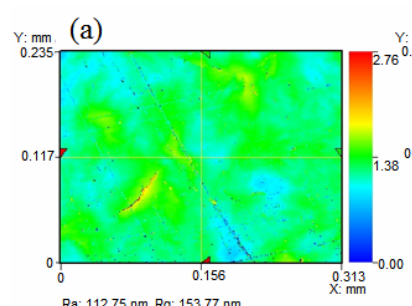

(b)

(c)
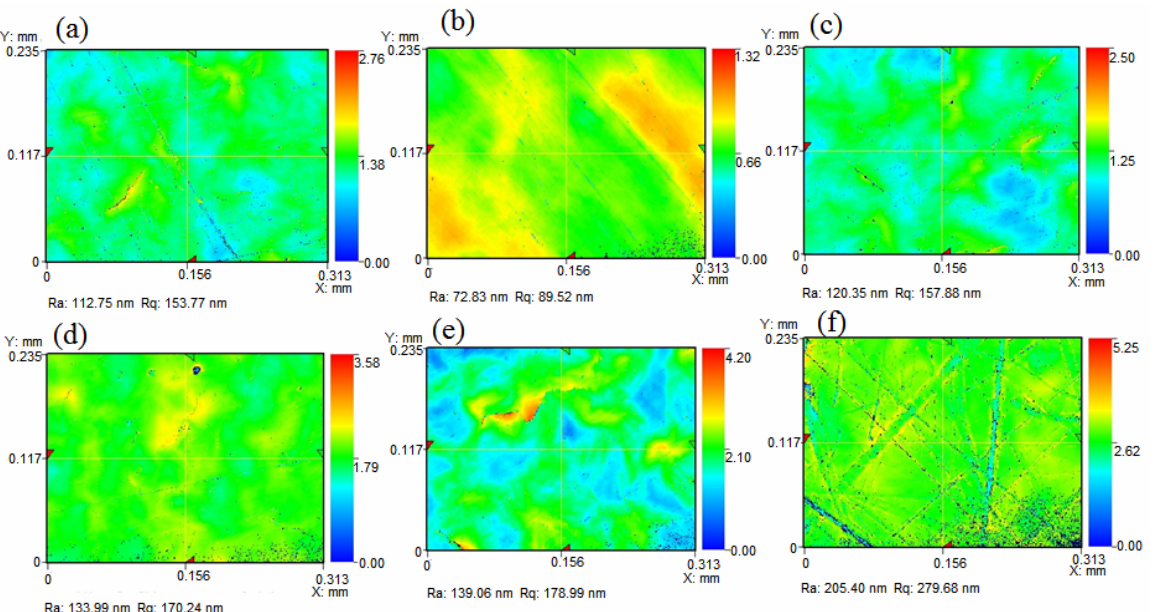

Ra: 120.35

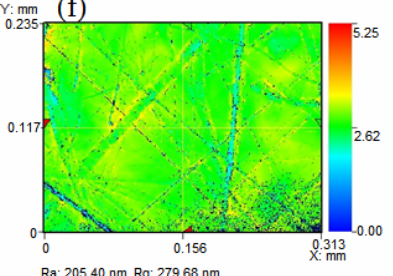

Fig. 8. 2D surface profile images on POM-C samples at 20x objective: (a) unirradiated; (b) 100 kGy; (c) $200 \mathrm{kGy}$; (d) $300 \mathrm{kGy}$; (e) $500 \mathrm{kGy}$ and (f) $700 \mathrm{kGy}$.

\section{CONCLUSION}

From the optical microscopic, tribometer, SEM, Raman spectroscopic and surface profiler observations, it can be concluded that $165 \mathrm{KeV}, 100 \mathrm{kGy}$ EB dose irradiation transferred the wear behavior of POM-C block from the abrasive wear, adhesive wear and scraping for the unirradiated surface to shallow and mild scraping for the irradiated surface due to well suited cross-linking, partial physical degradation, increased micro surface hardness, carbonization and formation of a small molecular substance. It also reduced the surface wear rate and average surface roughness with higher vibrational bands strength and micro surface hardness, which are ascertained from tribometer, surface profiler and Raman spectroscopic observations. Moreover, the $100 \mathrm{kGy}$ EB dose irradiated POM-C surface play as a self lubricating surface due to chemical free radicals formation.

Moreover, the electron beam irradiation condition (energy and dose) is the main parameter to reduce wear rate and chain scission depending on chemical and physical factors of POM-C surface.

\section{Acknowledgements}

This study was financially supported by Professor Dr. Heon-Ju Lee (Nuclear Fusion and Plasma Applications Laboratory, Department of Nuclear and Energy Engineering, Jeju National University, Republic of Korea) under Brain of Korea 21+ project. The authors also acknowledge the assistance of Dr. B.H. Choi and Professor K.H. Chung (Korea Accelerator and Plasma Research Association, Gangwon-do, South Korea) for invaluable directions in electron beam irradiation technique.

\section{References}

[1] Y. Duan, H. Li, L. Ye and X. Liu: "Study on the thermal degradation of polyoxymethylene by thermogravimetry-fourier transform infrared spectroscopy (TG-FTIR)." Journal of Applied Polymer Science, vol. 99, no. 6, pp. 3085-3092, 2006.[2] S. Raghu, S. Kilarkaje, G. Sanjeev, G.K. Nagaraja and H. Devendrappa: "Effect of electron beam irradiation on polymer electrolytes: Change in morphology, crystallinity, dielectric constant and AC conductivity with dose." Radiation Physics and Chemistry, vol. 98, no. January 2016, pp. 124-131, 2014. 
Study on Wear and Morphological Behavior of Electron Beam Dose Irradiated

[3] A. Turos, J. Jagielski, L. Slusarski and N.K. Madi: "Ion beam modification of surface properties of polyethylene." Vacuum, vol. 70, pp. 201-206, 2003.

[4] A. Abiona and a G. Osinkolu: "No Title." International Journal of Physical Sciences, vol. 5, no. 7, pp. 960-967, 2010.

[5] R.C. Ramola, S. Chandra, A. Negi, J.M.S. Rana, S. Annapoorni, R.G. Sonkawade, P.K. Kulriya and a.Srivastava: "Study of optical band gap, carbonaceous clusters and structuring in CR-39 and PET polymers irradiated by $100 \mathrm{MeV}$ O7+ ions." Physica B: Condensed Matter, vol. 404, no. 1, pp. 26-30, 2009.

[6] E. Reichmanis, C.W. Frank, J.H. O’Donnell and D.J.T. Hill: "Radiation Effects on Polymeric Materials.” Irradiation of Polymeric Materials, pp. 1-8, American Chemical Society (1993).

[7] J.H. O’Donnell: "Radiation Chemistry of Polymers." The Effects of Radiation on HighTechnology Polymers, pp. 1-13. ACS (1989).

[8] R. Gopal, M. Zuwei, S. Kaur and S. Ramakrishna: "Surface Modification and Application of Functionalized Polymer Nanofibers." Molecular Building Blocks for Nanotechnology, pp. 72 91. Springer New York, New York, NY (2007).

[9] Stephen C. Lapin: "Modification of Polymer Substrates using Electron Beam Induced Graft Copolymerization." Radtech Conference, no. 2, 2014.

[10] W. Schnabel: "Polymers and Electromagnetic Radiation." Wiley-VCH Verlag GmbH \& Co. KGaA, Weinheim, Germany, 2014.

[11] R. Deepalaxmi and V. Rajini: "Gamma and electron beam irradiation effects on SiR-EPDM blends."Journal of Radiation Research and Applied Sciences, vol. 7, no. 3, pp. 1-8, 2014.

[12] N. Tian, T. Li, X. Liu and W. Liu: "Effect of radiation on the friction-wear properties of polyetherketone with a cardo group." Journal of Applied Polymer Science, vol. 82, no. 4, pp. 962-967, 2001.

[13] X. Pei and Q. Wang: "Effect of Electron Radiation on the Tribological Properties of Polyimide.” Tribology Transactions, vol. 50, no. 2, pp. 268-272, 2007.

[14] K.A. Murray, J.E. Kennedy, B. Mcevoy, O. Vrain, D. Ryan, R. Cowman and C.L. Higginbothama: "Characterisation of the Surface and Structural Properties of Gamma Ray and Electron Beam Irradiated Low Density Polyethylene." International Journal of Material Science (IJMSCI), vol. 3, no. 1, pp. 1-8, 2013.

[15] T.T. Nguyen, C. Gobinet, J. Feru, S. Brassart-Pasco, M. Manfait and O. Piot: "Characterization of type I and IV collagens by Raman microspectroscopy: Identification of spectral markers of the dermoepidermal junction." Advances in Biomedical Spectroscopy, vol. 27, no. 5,-no. 6, pp. 421-427, 2012.

[16] C. Hirschl, M. Biebl-Rydlo, M. DeBiasio, W. Mühleisen, L. Neumaier, W. Scherf, G. Oreski, G. Eder, 20 B. Chernev, W. Schwab and M. Kraft: "Determining the degree of crosslinking of ethylene vinyl acetate photovoltaic module encapsulants-A comparative study." Solar Energy Materials and Solar Cells, vol. 116, pp. 203-218, 2013.

[17] H. Vaskova, D. Manas, M. Ovsik, M. Manas, and M. Stanek: "Microhardness of polyamide 12 after crosslinking due to beta radiation." International Journal of Mathematical Models and Methods in Applied Sciences, vol. 7, no. 1, pp. 83-90, 2013.

[18] C. Hirschl, L. Neumaier, G. Eder, and M. Kraft: "Raman Spectroscopic Determination of the Degree of Encapsulant Crosslinking in PV Modules." EU PVSEC Proceedings, no. SEPTEMBER 2013, pp. 3020-3025, 2013. 
[19] R. Hardis, J.L.P. Jessop, F.E. Peters, and M.R. Kessler: "Cure kinetics characterization and monitoring of an epoxy resin using DSC, Raman spectroscopy, and DEA." Composites Part A: Applied Science and Manufacturing, vol. 49, pp. 100-108, 2013.

[20] B.S. Chernev, C. Hirschl, and G.C. Eder: "Non-destructive determination of ethylene vinyl acetate cross-linking in photovoltaic (PV) modules by raman spectroscopy." Applied Spectroscopy, vol. 67, no. 11, pp. 1296-1301, 2013.

[21] H. Tadokoro, A. Kobayashi, Y. Kawaguchi, S. Sobajima, S. Murahashi, and Y. Matsui: "Normal Vibrations and Raman Spectrum of Polyoxymethylene." The Journal of Chemical Physics, vol. 35, no. 1, pp. 369, 1961. 